\title{
ON A REDUCTION PROCEDURE FOR HORN INEQUALITIES IN FINITE VON NEUMANN ALGEBRAS
}

\author{
BenoÎT COLlins AND KEN DYKeMA
}

\begin{abstract}
We consider the analogues of the Horn inequalities in finite von Neumann algebras, which concern the possible spectral distributions of sums $a+b$ of self-adjoint elements $a$ and $b$ in a finite von Neumann algebra. It is an open question whether all of these Horn inequalities must hold in all finite von Neumann algebras, and this is related to Connes' embedding problem. For each choice of integers $1 \leqslant r \leqslant n$, there is a set $T_{r}^{n}$ of Horn triples $(I, J, K)$ of $r$-tuples of integers, and the Horn inequalities are in one-to-one correspondence with $\cup_{1 \leqslant r \leqslant n} T_{r}^{n}$.

We consider a property $\mathrm{P}_{n}$, analogous to one introduced by Therianos and Thompson in the case of matrices, amounting to the existence of projections having certain properties relative to arbitrary flags, which guarantees that a given Horn inequality holds in all finite von Neumann algebras. It is an open question whether all Horn triples in $T_{r}^{n}$ have property $\mathrm{P}_{n}$. Certain triples in $T_{r}^{n}$ can be reduced to triples in $T_{r}^{n-1}$ by an operation we call TT-reduction. We show that property $\mathrm{P}_{n}$ holds for the original triple if property $\mathrm{P}_{n-1}$ holds for the reduced one.

A major part of this paper is devoted to showing that this operation of reduction preserves the value of the corresponding Littlewood-Richardson coefficients. We then characterize the TT-irreducible Horn triples in $T_{3}^{n}$, for arbitrary $n$, and for those LR-minimal ones (namely, those having Littlewood-Richardson coefficient equal to 1 ), we perform a construction of projections with respect to flags in arbitrary von Neumann algebras in order to prove property $\mathrm{P}_{n}$ for them. This shows that all LR-minimal triples in $\cup_{n \geqslant 3} T_{3}^{n}$ have property $\mathrm{P}_{n}$, and so that the corresponding Horn inequalities hold in all finite von Neumann algebras.
\end{abstract}

Mathematics subject classification (2000): 46L10.

Keywords and phrases: Horn inequality, finite von Neumann algebras.

\section{REFERENCES}

[1] H. BERCOVICI AND W.S. LI, Inequalities for eigenvalues of sums in a von Neumann algebra. Recent advances in operator theory and related topics (Szeged, 1999), Oper. Theory Adv. Appl., 127 (2001), Birkhäuser, Basel, 113-126.

[2] H. Bercovici And W.S. Li, Eigenvalue inequalities in an embeddable factor, Proc. Amer. Math. Soc., 134 (2006), 75-80.

[3] A. S. BUCH, Littlewood-Richardson Calculator, programs written in C and maple code, http://www.math.rutgers.edu/ asbuch/lrcalc/

[4] B. Collins, K. Dykema, A linearization of Connes' embedding problem, New York J. Math. 14 (2008), 617-641.

[5] W. Fulton, Young Tableaux, Cambridge University Press (Cambridge, U.K.), 1997.

[6] W. Fulton, Eigenvalues, invariant factors, highest weights and Schubert calculus, Bull. Amer. Math. Soc. (N.S.), 37 (2000), 209-249. 
[7] A. Horn Eigenvalues of sums of Hermitian matrices, Pacific J. Math., 12 (1962), 225-241.

[8] R. C. ThOMPSON AND S. Therianos, On a Construction of B. P. Zwahlen, Linear and Multilinear Algebra, 1 (1974), 309-325.

[9] B. P. Zwahlen, Über die Eigenwerte der Summe zweier selbstadjungierter Operatoren, Comment. math. Helv., 40 (1966), 81-116.

[10] H. Bercovici, B. Collins, K. Dykema, W. S. Li And D. Timotin, Intersections of Schubert varieties and eigenvalue inequalities in an arbitrary finite factor, arxiv:0805.4817 